\title{
Kasuistiken
}

Rechtsmedizin 2021 · 31:538-544 https://doi.org/10.1007/s00194-021-00466-9

Angenommen: 13. Januar 2021

Online publiziert: 17. Februar 2021

๑c Der/die Autor(en) 2021

\author{
V. Thoma' · V. Auwärter ${ }^{2} \cdot$ A. Thierauf-Emberger ${ }^{1} \cdot$ S. Pollak ${ }^{1}$ \\ ${ }^{1}$ Institut für Rechtsmedizin, Medizinische Fakultät, Universität Freiburg, Freiburg, Deutschland \\ ${ }^{2}$ Forensische Toxikologie, Institut für Rechtsmedizin, Medizinische Fakultät, Universität Freiburg, Freiburg, \\ Deutschland
}

\section{Unvollendet gebliebener Versuch eines erweiterten Suizids durch Stromeinwirkung in der Badewanne}

\section{Einleitung}

Der erweiterte Suizid, auch HomizidSuizid genannt, ist ein schwerwiegendes Ereignis, bei dem eine zur Selbsttötung entschlossene Person mindestens einen weiteren Menschen ohne dessen Zustimmung bzw. gegen dessen Willen mit in den Tod nimmt [1]. Fremd- und Selbsttötung folgen charakteristischerweise in kurzer zeitlicher Abfolge aufeinander. Angaben zu konkreten Zeitspannen variieren in der vorhandenen Literatur jedoch erheblich. Für manche Autoren ist das Zeitkriterium selbst dann noch erfüllt, wenn zwischen Homizid und Suizid bis zu 3 Monate liegen [2].

In den allermeisten Fällen handelt es sich bei den Betroffenen um enge Angehörige wie Familienmitglieder oder Intimpartner [1,3], weshalb diese Fälle anfangs auch als „Familienmorde“ bezeichnet wurden. Abwandlungen, Erweiterungen und Überschneidungen mit anderen Formulierungen finden sich in früheren Publikationen [4]. Auf die daraus resultierende begriffliche Ungenauigkeit verwies Foerster [4]; er schlug daher die Verwendung des neutralen, auf rein formalen Kriterien basierenden Begriffs „Tötung mit nachfolgendem Suizid(versuch)“vor.

Die Datenlage zu allen Formen einer Tötung mit anschließend versuchter/ vollendeter Selbsttötung ist relativ spärlich [4]. Im Münchner Sektionsgut des Jahres 2010 wurden unter insgesamt 376 Selbsttötungen 10 Fälle eines erweiterten Suizides (2,6\%) erfasst [5]. Eine Un- tersuchung des rechtsmedizinischen Instituts der Charité und des Landesinstituts für gerichtliche und soziale Medizin in Berlin ergab im Zeitraum von 2005 bis 2013 insgesamt 17 Fälle [6]. Die von Coid 1983 publizierte Inzidenzrate von 0,1-0,4/100.000 Ew. stützt sich auf 17 Studien aus den Jahren 1900-1979 und umfasst 10 Nationen. Über Ländergrenzen hinweg war die Inzidenzrate relativ ähnlich und konstant [7]. In einem neueren Review aus dem Jahr 2009 kommt Eliason [8] zu dem Fazit, dass der erweiterte Suizid mit einer Inzidenz von etwa 0,2-0,3/100.000 Ew. und Jahr ein relativ seltenes Ereignis ist. Im Übrigen sind exakte Zahlen zur Inzidenz nur schwer zu eruieren, da der Begriff „erweiterter Suizid" nicht einheitlich definiert ist und die einschlägigen Fälle in den offiziellen Statistiken u. U. separat als Suizide und Tötungsdelikte erfasst werden [9].

Im Hinblick auf die Zahl der von einem Täter getöteten Menschen stellten Barraclough und Harris [3] fest, dass in $80 \%$ der Fälle jeweils ein Opfer betroffen war. Die Autoren untersuchten alle Homizide in England und Wales von 1988 bis 1992 und fanden heraus, dass $19 \%$ aller Kindstötungen im Rahmen eines erweiterten Suizids begangen wurden. Nach der Auswertung von Byard ist der Anteil weiblicher Täter höher, wenn die eigenen Kinder als Opfer involviert waren [9].

Im Folgenden wird ein Fall von unvollendet gebliebenem Homizid-Suizid vorgestellt, der in mehrfacher Hinsicht - Einbeziehung eines Kindes im Schulal- ter, Verwendung eines Elektrogeräts als Tatmittel, Begehung des versuchten Filizids in einer Badewanne, folgenloses Überleben beider Beteiligter - ganz ungewöhnlich ist und in der konkret vorgelegenen Konstellation bisher noch nicht veröffentlicht wurde.

\section{Kasuistik}

Hergang und Umstände des Falles

Eine 36 Jahre alte Frau wurde am späten Abend, gegen 22.30 Uhr, von ihrem Vater nackt und in benommenem $\mathrm{Zu}$ stand in der mit Wasser gefüllten Badewanne aufgefunden. Zuvor hatte die Frau nach eigenen Angaben 8 „Schlaftabletten“ eingenommen und eine größere Menge Rotwein getrunken. Anschließend habe sie sich mit ihrer 7-jährigen, ebenfalls unbekleideten Tochter in die Badewanne begeben und einen laufenden Föhn in das Badewasser eingebracht. Zusätze mit Einfluss auf die elektrische Leitfähigkeit seien dem Badewasser nicht beigegeben worden. Der Tochter sei es gelungen, aus der Badewanne zu steigen und die Großeltern zu verständigen. Als der Großvater versucht habe, seine Tochter aus der Badewanne zu ziehen, habe er einen Stromfluss verspürt, und seine Hände hätten sich verkrampft. Anschließend habe er den noch eingesteckten und laufenden Föhn im Wasser bemerkt und diesen aus der Wanne entfernt (• Abb. 1). Über die ursprüngliche Position des Föhns innerhalb der Bade- 


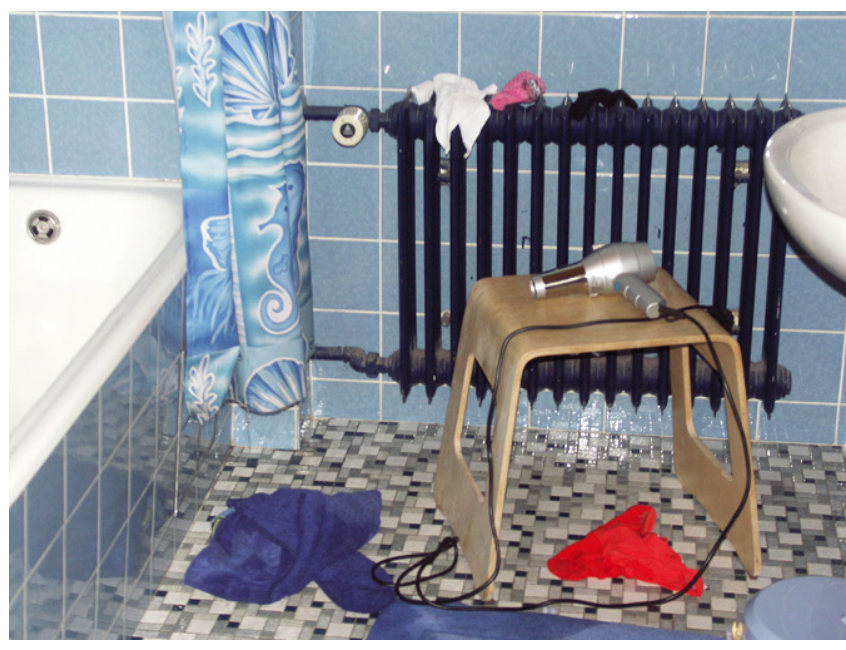

Abb. 1 A Ereignisort beim Eintreffen der Polizei. Der noch eingesteckte Föhn befindet sich nicht mehr in der (mittlerweile geleerten) Badewanne, sondern daneben auf dem Badezimmerhocker

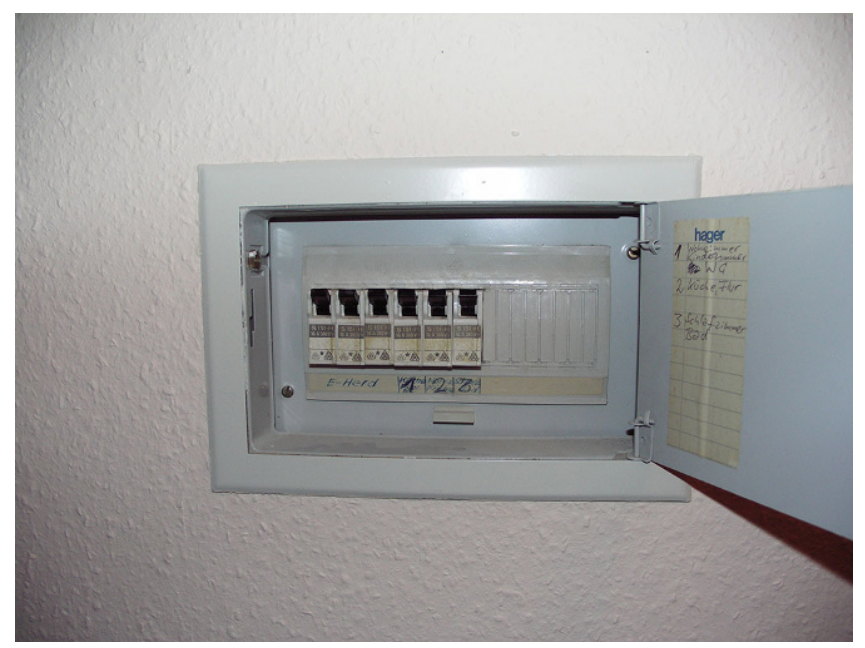

Abb. $2 \Delta$ Leitungsschutzschalter der Wohnung, in der sich der versuchte Homizid-Suizid ereignet hat. Ein Fehlerstrom-Schutzschalter ist nicht vorhanden. Bei keinem der Schutzschalter ist es zur Auslösung des Abschaltmechanismus gekommen. Die Nr. 3 bezeichnet den Leitungsschutzschalter für das Schlafzimmer und Bad wanne liegen keine Erkenntnisse vor. $\mathrm{Zu}$ sammen mit seiner Ehefrau sei es ihm gelungen, die Tochter aus der Wanne zu ziehen.

Die 36-jährige Frau und ihre 7-jährige Tochter wurden vorsorglich zur Beobachtung in ein Krankenhaus eingeliefert. Auch der Vater bzw. Großvater, der seit 10 Jahren an Herzrhythmusstörungen litt, wurde nach dem Stromschlag wegen neuerlich aufgetretener Rhythmusstörungen stationär aufgenommen.

Laut Angaben der behandelnden Ärzte wies die aus der Badewanne geborgene Frau äußerlich keine sichtbaren Verletzungen auf. Konzentrationsbestimmungen der Gesamtkreatinkinase (CK) und des Isoenzyms vom Myokardtyp (CK$\mathrm{MB}$ ) wurden von klinischer Seite nicht veranlasst. Auch ihre 7-jährige Tochter war unverletzt; sie gab an, keinen Stromschlag bemerkt zu haben. Ärztlicherseits wurden keine Strommarken festgestellt. Mutter und Kind waren kreislaufstabil und wiesen keine Herzrhythmusstörungen auf.

Eine Überprüfung des Sicherungskastens in der Wohnung ergab, dass keine der Sicherungen angesprochen hatte (• Abb. 2). Ein Fehlerstrom(FI)-Schutzschalter war nicht installiert.

Im Wohnzimmer wurde am Fußende der Couch und auf dem Fußboden erbrochener, nach Wein riechender Mageninhalt vorgefunden.
Konkrete Suizidabsichten hatte die 36jährige Frau vor der Tat nicht geäußert. Sie habe sich lediglich in den vergangenen 2 Wochen in ihre Wohnung zurückgezogen und kaum Kontakt zur Familie gesucht. Ein Abschiedsbrief konnte nicht aufgefunden werden.

Gegenüber der Polizei räumte die Frau ein, dass sie ihr Leben und das ihrer 7-jährigen Tochter habe beenden wollen. Sie war alleinerziehend. Zum Vater ihrer Tochter bestand keine Beziehung mehr.

\section{Chemisch-toxikologische Untersuchungen}

Zur Tatzeit um 22.30 Uhr betrug die rückgerechnete Blutalkoholkonzentration bei der 36-jährigen Frau zwischen 0,49\%o und 1,2\%o. Ein immunologisches Screening aufBetäubungsmittel verlief negativ. In einer nach der Tat entnommenen Blutprobe wurde Zolpidem, eine benzodiazepinähnliche Substanz mit sedativ-hypnotischer Wirkung, in einer Konzentration von $8 \mathrm{ng} / \mathrm{ml}$ nachgewiesen. Auf die Vorfallzeit extrapoliert, ergab sich eine maximale Konzentration von $80 \mathrm{ng} / \mathrm{ml}$, was bedeutet, dass der therapeutische Bereich (80-200 ng/ml) eben erreicht wurde.

Eine am Ereignisort vorgefundene Tablette wurde auf ihre Inhaltsstoffe untersucht. Dabei konnten keine pharmakologisch wirksamen Inhaltsstoffe, insbesondere kein Zolpidem, nachgewiesen wer- den. Das toxikologische Gutachten kam zu der Beurteilung, dass die Angaben der Beschuldigten (8 Tabletten und Rotwein in beträchtlicher Menge) nicht widerlegt werden können, da es sich bei den zugeführten Tabletten zumindest teilweise um ein wirkstofffreies Präparat gehandelt haben könnte und ein Teil des getrunkenen Rotweins (und vermutlich auch der eingenommenen Tabletten) erbrochen wurde.

\section{Elektrotechnische Untersuchungen}

Im vorgestellten Fall bestätigte das Sachverständigengutachten (Dr. Löhle \& Simon, Freiburg) die Funktionstüchtigkeit des Haarföhns. Versuche mit dem sichergestellten Gerät in einem Wasserbecken ergaben, dass die gemessenen Stromstärken bei voller Heizleistung (1500 W) - je nach Positionierung innerhalb der Badewanne - erheblich variierten. Stark vereinfacht sind grundsätzlich folgende 3 Szenarien denkbar (bei konstanter Lage des Föhns):

1. Haarföhn in Körperkontakt bei gleichzeitigem Kontakt mit einem geerdeten Teil (etwa Abfluss, Überlauf, Wasserarmatur) ergab Stromstärken zwischen 90 und $130 \mathrm{~mA}$.

2. Haarföhn möglichst weit vom geerdeten Abfluss entfernt; hierbei konnten Stromstärken gegen Erde 
von $40-80 \mathrm{~mA}$ und im Wasser von 3-10 mA gemessen werden.

3. Haarföhn direkt über dem Abfluss; dabei betrugen die gemessenen Stromstärken $<1 \mathrm{~mA}$.

Es ist jedoch $\mathrm{zu}$ beachten, dass ein frei in der Badewanne liegender Haarföhn mit eingeschaltetem Gebläse seine Position strömungsbedingt ändert, sodass sich in der Praxis zwangsläufig unterschiedliche Konstellationen einstellen. Ein eingesteckter Föhn innerhalb der Badewanne kann somit eine konkret lebensbedrohliche Durchströmung zur Folge haben. Dies wird durch zahlreiche Fallbeispiele aus der rechtsmedizinischen Literatur und Praxis bestätigt.

\section{Psychopathologischer Befund und psychiatrisches Gutachten}

Bei der Exploration knapp 11 Wochen nach der Tat erschien die 36-jährige Frau leicht affektlabil mit verzweifelter Grundstimmung. Ihr Selbstwertgefühl sei massiv vermindert gewesen, und sie habe deutliche Schuldgefühle wegen des inkriminierten Vorfalls geäußert.

Bei Beleuchtung des Lebenslaufs der Frau sei die negative Beziehung zu ihrem Vater deutlich geworden. Bekannte und Freunde hätten ihr keine berufliche Karriere zugetraut, weshalb sie viel gegrübelt und häufig depressive Phasen gehabt habe. Sie habe sich deshalb auch in psychotherapeutischer Behandlung befunden.

Der Vater ihres Kindes habe erhebliche Alkohol- und Drogenprobleme gehabt. Auch sie selbst sei kurzzeitig in der "Szene“ unterwegs gewesen. Nach 5-jähriger Beziehung sei die Trennung von ihrem Partner erfolgt. Bis etwa zum 2. Lebensjahr des Kindes habe sich die Frau in einem Mutter-Kind-Projekt befunden. Später sei es zu Spannungen zwischen ihr und dem Kindsvater gekommen, da die Tochter nach Kontakten mit dem Vater „komische Äußerungen“ gemacht habe, weshalb der Verdacht eines sexuellen Missbrauchs der Tochter aufgekommen sei. Ein Anwalt habe ihr von einer diesbezüglichen Anzeige abgeraten. Ihre psychischen Probleme seien verstärkt aufgetreten, als der Kindsvater versucht habe, die Tochter gegen die

Rechtsmedizin 2021 · 31:538-544 https://doi.org/10.1007/s00194-021-00466-9

(c) Der/die Autor(en) 2021

\section{Thoma · V. Auwärter · A. Thierauf-Emberger · S. Pollak}

\section{Unvollendet gebliebener Versuch eines erweiterten Suizids durch Stromeinwirkung in der Badewanne}

\section{Zusammenfassung}

Während sich heutzutage in westlichen Ländern erweiterte Suizide ganz überwiegend in (gescheiterten) Partnerbeziehungen ereignen, stellen Kindstötungen mit nachfolgendem Suizid eines Elternteils ein seltenes Ereignis dar. Vorgestellt wird der ungewöhnliche Fall eines versuchten HomizidSuizides einer 36-jährigen Frau, die durch das Einbringen eines eingeschalteten Föhns in das Badewasser beabsichtigte, sich und ihre 7 Jahre alte Tochter zu töten. Während sich das Kind selbstständig aus der Wanne befreien und die Großeltern informieren konnte, wurde die Frau in benommenem Zustand von ihrem Vater vorgefunden. Beim Versuch, seine Tochter aus der Badewanne zu bergen, habe er einen Stromfluss verspürt. Bemerkenswerterweise haben alle 3 Personen ohne gesundheitlichen Schaden überlebt; sie wiesen keine Strommarken auf. Die ungewöhnliche Fallkonstellation, die elektrotechnischen Aspekte und die psychiatrische Vorgeschichte der Täterin, die zum Tatzeitpunkt unter dem Einfluss von Alkohol und eines sedierenden Mittels stand, werden unter Berücksichtigung der einschlägigen Literatur diskutiert.

\section{Schlüsselwörter}

(Unvollendeter) Homizid-Suizid · Filizid . Stromschlag · Forensische Toxikologie . Forensische Psychiatrie

\section{Attempted homicide-suicide by means of electrocution in the bathtub}

\begin{abstract}
In Western societies, currently most homicidesuicides are committed in problematic partnerships. In contrast, filicides followed by the suicide of a parent have become rare incidents. The present paper describes an uncommon case of the latter kind. A 36year-old woman tried to kill her 7-yearold daughter and herself in the bathtub by placing a switched on hairdryer into the water. The girl could free herself without help, whereas her mother remained lying in the bathtub together with the still working electrical device. She was recovered by her father who had meanwhile been informed by the unharmed girl. While attempting
\end{abstract}

to pull his daughter out of the bathtub he felt an electric current pass through his body. Surprisingly, all three persons survived without bodily harm and did not show any electrical current marks. The unique case constellation, the electrotechnical aspects and the psychiatric history of the perpetrator, who had been under the influence of alcohol and a sedative agent, are discussed with reference to the pertinent literature.

\section{Keywords}

(Attempted) homicide-suicide - Filicide . Electrocution · Forensic toxicology · Forensic psychiatry
Mutter aufzuhetzen. In diesem Zusammenhang berichtete sie von Grübelzwängen, innerer Anspannung, Verzweiflung, Ratlosigkeit sowie Depressionen mit Erschöpfung und Gereiztheit.

Einige Zeit vor der gegenständlichen Tat sei eine neue partnerschaftliche Beziehung zerbrochen, was sie schwer belastet habe. Zudem berichtete die Beschuldigte über Probleme mit ihrer eigenen Mutter und mit dem Arbeitgeber. Sie habe starke Schlafstörungen entwickelt und „mit dem Leben Schluss machen“ wollen. Grund hierfür sei gewesen, dass sie zu wenig Hilfe angenommen und nicht eingesehen habe, dass sie überfordert und überlastet gewesen sei. Sie habe viele „Baustellen“ in ihrem Leben gehabt, die sie einfach nicht mehr habe bewältigen können.

Der psychiatrische Gutachter stellte die Diagnose einer rezidivierenden, (mittelschweren) depressiven Episode in Verbindung mit einer dysthymen und emotional labilen Persönlichkeit. Maladaptive Verarbeitungsmechanismen und schwierige Lebensumstände hätten die depressiven Phasen begünstigt. $\mathrm{Zu}$ - 
nehmende psychosoziale Belastungen (multiple Beziehungskonflikte, erhebliche Selbstunsicherheit - auch in der Rolle als Mutter, materielle Probleme, sozialer Rückzug, schwere psychovegetative Erschöpfung, Grübelzwänge, Kontaktstörung zu Familienangehörigen, Freunden und Bekannten, Arbeitslosigkeit, vielfältige Selbstzweifel und Enttäuschungen) hätten schließlich $\mathrm{zu}$ einem präsuizidalen Syndrom geführt, das sich durch verstärkte und gleichzeitig gehemmte (Selbst-)Aggressionen geäußert habe.

Als Hauptmotiv für den Versuch eines erweiterten Suizids wird im Gutachten genannt, dass die Frau auch für ihr Kind „nur noch negative Zukunftsperspektiven“ gesehen habe. Bei einem alleinigen Suizid der Mutter hätte der Vater „ungeschützten Zugriff“ auf seine Tochter gehabt. Insofern sei die Mitnahme des eigenen Kindes von der Beschuldigten als ein "rettender Akt" aufgefasst worden.

Die 36 Jahre alte Frau wurde wegen versuchten Totschlags zu einer mittlerweile rechtskräftigen Freiheitsstrafe von 15 Monaten verurteilt; die Vollstreckung ist auf Bewährung ausgesetzt worden.

\section{Diskussion}

Überlebte Stromeinwirkungen in der Badewanne sind selten $[10,11]$ und fanden bisher in der wissenschaftlichen Literatur nur wenig Beachtung. Entsprechend rar sind publizierte Kasuistiken.

Im vorgestellten Fall versuchte eine 36 Jahre alte Frau, sich selbst und ihre 7-jährige Tochter in einer mit Wasser gefüllten Badewanne durch das Einbringen eines eingeschalteten Föhns zu töten. Nach den Ergebnissen der chemisch-toxikologischen Untersuchungen lag bei der Täterin eine mäßige Alkoholisierung vor. Im Blut wurde zudem eine therapeutische Konzentration des Schlafmittels Zolpidem nachgewiesen.

Pollak [12] und Byard [9] kamen zu dem Ergebnis, dass weibliche Täter weniger oft „harte“ Tötungsmethoden anwenden als Männer. Während männliche Täter nicht nur ihre Kinder, sondern auch ihre Lebenspartnerinnen töten man spricht in solchen Fällen von „family annihilators“ -, ist das Töten des
Ehepartners oder von nichtfamilienzugehörigen Kindern für weibliche Täter untypisch [13].

Von 209 in den USA begangenen Homizid-Suiziden aus den Jahren 2003 und 2004 [14] wurden in 23 Fällen insgesamt 30 Kinder unter 15 Jahren getötet. In fast drei Viertel der Fälle wurde die Tat von einem Elternteil begangen. Mögliche Motive waren juristische Auseinandersetzungen, finanzielle und berufliche Schwierigkeiten sowie Beziehungsprobleme. Fast jeder 10. Täter hatte eine Drogen- bzw. Alkoholanamnese; $29 \%$ wurden nach der Tat positiv auf Drogen oder Alkohol getestet. Ähnlich hohe Alkoholisierungsraten wurden bereits in der Vergangenheit beschrieben (27\% [15], 31\% [16]). Eine akute psychische Erkrankung lag bei $11 \%$ der Täter vor; 8,6\% litten an einer Depression, und bei 7,2\% waren aus der Vergangenheit psychische Probleme bekannt.

Depressive Störungen sind bei Tätern eines erweiterten Suizids weit häufiger als bei einem alleinigen Homizid [9, 17]. Friedman et al. [18] konnten zeigen, dass mehr als $80 \%$ der Eltern, die einen Filizid-Suizid begangen haben, psychiatrische Probleme hatten. An Depressionen litten $57 \%$, wobei mehrheitlich Frauen betroffen waren (70\% vs. $50 \%)$.

In einer Fallserie [19] wurden 12 Frauen nach einer (versuchten) erweiterten Selbsttötung unter psychiatrischen und kriminologischen Gesichtspunkten miteinander verglichen. Während eine der Frauen nach der Tötung ihres Kindes durch Suizid starb, überlebten die anderen Mütter. Vier Probandinnen litten an Schizophrenie, 4 weitere an einer endogenen Depression, eine an einer psychogenen Panikreaktion, und 3 wiesen Persönlichkeitsstörungen auf. Schuldunfähig waren alle Frauen mit Schizophrenie, 3 mit einer Depression (die 4. Depressive hatte sich suizidiert) und eine Täterin mit einer Persönlichkeitsstörung in Kombination mit Alkoholmissbrauch und Polytoxikomanie. Die Übrigen waren schuldfähig bzw. vermindert schuldfähig.

Hellen et al. [20] berufen sich auf eine Untersuchung von Fishbain et al. aus dem Jahr 1986 [21], wonach intendierte Homizid-Suizide von Frauen nicht sel- ten von diesen überlebt werden, was u. a. darin begründet sei, dass weibliche Täter weniger aggressive Suizidmethoden anwenden würden. Dies führe vermutlich dazu, dass die Taten wegen des unvollendet gebliebenen Suizids in den Statistiken nicht als Homizid-Suizide registriert werden.

Resnick untersuchte 131 Fälle von Filiziden und schlug 1969 eine Klassifikation nach der Motivlage vor [22]:

1. Altruistischer Filizid: Fast die Hälfte $(49 \%)$ der von Resnick untersuchten Fälle entfiel auf explizit (pseudo-)altruistische Motive. Dabei steht das Töten „aus Liebe“ im Vordergrund. Das Kind soll „erlöst“ werden: entweder um es nach erfolgter Selbsttötung des Elternteils vor dem Alleinsein zu bewahren oder um es von seinen (realen oder imaginären) Leiden bzw. Bedrohungen zu befreien. Dementsprechend beschrieb Adelson [23], dass Täter nach einer Kindstötung häufiger Suizid begehen, als dies nach Tötung von erwachsenen Opfern der Fall ist. Insbesondere bei versuchten, aber unvollendet gebliebenen Homizid-Suiziden ist aus rechtlicher Sicht zu klären, ob im konkreten Fall altruistische Motive als vorgeschobene Schutzbehauptung der überlebenden Täter/-innen angegeben werden, um die Tat zu begründen bzw. zu entschuldigen (Foerster [4]).

2. Akut-psychotischer Filizid: Kindstötung unter dem Einfluss von Halluzinationen, Epilepsie oder Delir (21\%).

3. Filizid eines ungewollten Kindes (14\%).

4. Unbeabsichtigter Filizid: Kindesmisshandlung mit tödlichem Ausgang, der nicht intendiert war (12\%).

5. Filizid aus Rache am Partner: Tötung des gemeinsamen Kindes, um dem Partner bewusst Leid zuzufügen (4\%).

Resnick zufolge kommt es nach einem Filizid aus (pseudo-)altruistischen Motiven bzw. unter akuten psychotischen Einflüssen bei den Tätern bzw. Täterinnen häufig zu einem sofortigen Abfall der Anspannung, was eine Erklärung für das Scheitern des geplant gewesenen Suizids sein kann.

Ab Mitte der 70er-Jahre des letzten Jahrhunderts wurde ein bemerkenswerter Anstieg der Elektrotodesfälle in der Badewanne verzeichnet [24, 25]. Im Zeit- 
raum von 1995 bis 1999 ereigneten sich in Berlin 41 Fälle von Stromtod in der Badewanne [24]. Von den Opfern waren $54 \%$ Frauen. Ein Überwiegen des weiblichen Geschlechts wurde bereits von Bonte et al. [25] mit einem Geschlechterverhältnis von 3:1 beschrieben (bei einer Gesamtfallzahl von 48).

Im Berliner Untersuchungsgut [24] wurden 30 Stromtodesfälle in der Badewanne (75\%) als Suizide klassifiziert. In den 48 von Bonte et al. [25] untersuchten Todesfällen handelte es sich allerdings lediglich in 23 Fällen um eine (angenommene) Selbsttötung (48\%). Lawrence et al. [26] berichteten, dass von 5000 ausgewerteten Suiziden nur 3 auf einen Stromtod zurückzuführen waren.

In $75 \%$ aller von Bockholdt und Schneider [24] beschriebenen Elektrotodesfälle in der Badewanne fungierte ein Haarföhn als Stromquelle. In 10 Fällen war der Föhn noch bei der Auffindung in Betrieb. Auch im Kollektiv von Bonte et al. [25] waren drei Viertel der Fälle durch Haartrockner verursacht.

Eindeutige Strommarken wurden im Untersuchungsgut von Bockholdt und Schneider [24] nur in 16 von 41 Fällen festgestellt. Dass bei Stromtodesfällen in der Badewanne deutlich seltener Strommarken festzustellen sind als bei solchen außerhalb des Wassers, führten Bonte et al. [25] darauf zurück, dass Badewasser als Elektrode dienen kann, also kein direkter Kontakt zwischen der Haut und einem metallischen Leiter nötig ist. Nicht selten führt das Weiterlaufen des Haarföhns zu einer überkritischen Erwärmung des Badewassers mit Ausbildung von prä- und/oder postmortalen Verbrühungen (Literatur: [27]).

Dass eine elektrische Durchströmung in der mit Wasser gefüllten Badewanne nicht zwingend zum Tod führen muss, belegt ein von Rothschild et al. [28] beschriebener Fall. Hierbei hatte ein 60 Jahre alter Suizident versucht, sich durch das Einbringen von 2 Elektrogeräten (Mixer und Bohrmaschine) in das Badewasser das Leben zu nehmen. Der Wannenkörper war nicht geerdet. Die Sicherungen hatten zu keiner Unterbrechung des Stromkreises geführt.

Von einschreitenden Helfern wurden Fälle berichtet, in denen beim Griff in das
Badewasser zwar ein Kribbeln verspürt wurde, aber keine (lebens)gefährlichen Situationen aufgetreten sind. Bonte et al. [25] berichteten 2 Fälle, in denen Helfer beim Griff in das Badewasser ein Kribbeln verspürt haben. Im zweiten Fall haben sich ein Mann und ein Kind in einer mit Wasser gefüllten Badewanne befunden. Während der Mann durch Stromschlag aufgrund eines in das Wassergefallenen Föhns starb, hat das Kind folgenlos überlebt.

Experimentell wurden bei nichtgeerdeten Badewannen lediglich in unmittelbarer Nähe der Stromquelle relativ große Potenzialdifferenzen gemessen [29]. Messungen ohne unmittelbaren Kontakt zum Elektrogerät ergaben im Bereich des Herzens eine Stromstärke von $55 \mathrm{~mA}$ [30]. Bei Erdung der Wanne wurde am Herz eine Stromstärke von $87 \mathrm{~mA}$ gemessen.

Bei ganzheitlicher Betrachtung lässt sich der vorgestellte Fall hinsichtlich seiner Motivlage als versuchter Homizid-Suizid aus (pseudo-)altruistischer Gesinnung klassifizieren. Es handelt sich damit um die nach Resnick [22] häufigste Motivkategorie unter den Filizid(versuch)en. Die Täterin habe nach eigenen Angaben ihre Tochter vor einer negativen Zukunftsperspektive und vor dem Einfluss des Kindsvaters schützen wollen. Die Tat habe sie „aus freien Stücken“, mit der Absicht, ihr Kind zu „retten“, geplant. Disponierend für die versuchte Selbsttötung waren laut psychiatrischem Gutachten wiederkehrende depressive Episoden in Verbindung mit zunehmenden psychosozialen Belastungen.

Eine weitere Besonderheit des vorgestellten Falls ist das Alter des Kindes, das in den erweiterten Suizid einbezogen werden sollte. Aus epidemiologischen Studien ist bekannt, dass kindliche Opfer von Homizid-Suiziden mehrheitlich jünger als 5 Jahre alt sind [31]. Deutlich seltener werden ältere Kinder oder Jugendliche im Rahmen von erweiterten Suiziden getötet. Aus dem Untersuchungsgut des Freiburger Instituts für Rechtsmedizin ist ein vollendeter erweiterter Suizid bekannt, bei dem ein 49 Jahre alter Mann seine 6 Jahre alte Tochter durch scharfe Gewalt (Messer, Hackmesser) getötet hat.

\section{Fazit für die Praxis}

- Die vorgestellte Kasuistik ist ein seltenes Beispiel für einen versuchten Homizid-Suizid, bei dem ein Kind im Schulalter in die Tat einbezogen wurde. Heutzutage ereignen sich erweiterte Suizide ganz überwiegend in (gescheiterten) Partnerbeziehungen.

- Die gewählte, aber unvollendet gebliebene Tötungsmethode (Einbringen eines Elektrogeräts in die mit Wasser gefüllte Badewanne) ist in der konkreten Konstellation bei HomizidSuiziden bisher nicht Gegenstand einer Veröffentlichung gewesen.

- Als Besonderheit ist hervorzuheben, dass alle 3 Personen (Mutter, Kind, Großvater), die einer elektrischen Durchströmung ausgesetzt waren, ohne gesundheitlichen Schaden überlebt haben. Dies lässt sich u.a. damit erklären, dass die in einer wassergefüllten Badewanne einwirkende Stromstärke ganz wesentlich von der Position des Haarföhns und einem etwaigen Körperkontakt mit geerdeten Teilen abhängt. Es empfiehlt sich daher, in ähnlichen Fällen elektrotechnische Aspekte bei der Tataufklärung zu berücksichtigen.

- Insbesondere bei versuchten, aber unvollendet gebliebenen HomizidSuiziden ist des Weiteren aus rechtlicher Sicht zu klären, ob altruistische Motive als vorgeschobene Schutzbehauptung der überlebenden Täter/innen angegeben werden, um die Tat zu begründen bzw. zu entschuldigen.

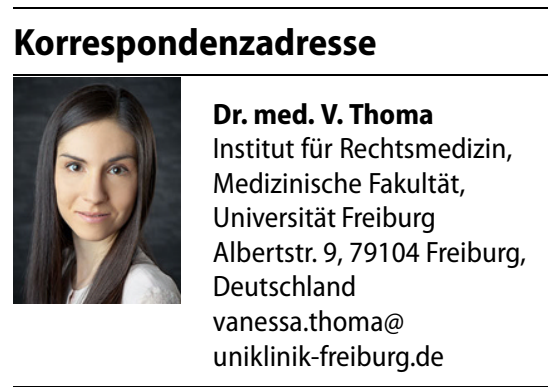

Funding. Open Access funding enabled and organized by Projekt DEAL. 


\section{Einhaltung ethischer Richtlinien}

Interessenkonflikt. V. Thoma, V. Auwärter, A. Thierauf-Emberger und S. Pollak geben an, dass kein Interessenkonflikt besteht.

Für diesen Beitrag wurden von den Autoren keine Studien an Menschen oder Tieren durchgeführt.

Open Access. Dieser Artikel wird unter der Creative Commons Namensnennung 4.0 International Lizenz veröffentlicht, welche die Nutzung, Vervielfältigung, Bearbeitung, Verbreitung und Wiedergabe in jeglichem Medium und Format erlaubt, sofern Sie den/die ursprünglichen Autor(en) und die Quelle ordnungsgemäß nennen, einen Link zur Creative Commons Lizenz beifügen und angeben, ob Änderungen vorgenommen wurden.

Die in diesem Artikel enthaltenen Bilder und sonstiges Drittmaterial unterliegen ebenfalls der genannten Creative Commons Lizenz, sofern sich aus der Abbildungslegende nichts anderes ergibt. Sofern das betreffende Material nicht unter der genannten Creative Commons Lizenz steht und die betreffende Handlung nicht nach gesetzlichen Vorschriften erlaubt ist, ist für die oben aufgeführten Weiterverwendungen des $\mathrm{Ma}$ terials die Einwilligung des jeweiligen Rechteinhabers einzuholen.

Weitere Details zur Lizenz entnehmen Sie bitte der Lizenzinformation auf http://creativecommons.org/ licenses/by/4.0/deed.de.

\section{Literatur}

1. Faller-Marquardt M, Pollak S (2006) Erweiterter Suizid mit Tötung von 5 Familienangehörigen aus 3 Generationen. In: Kauert G, Mebs D, Schmidt P (Hrsg) Kausalität. Forensische Medizin, Toxikologie, Biologie, Biomechanik und Recht. Hansjürgen Bratzke zum 60. Geburtstag. Berliner Wissenschafts-Verlag, Berlin, S43-53

2. Allen NH (1983) Homicide followed by suicide: Los Angeles, 1970-1979. Suicide Life Threat Behav 13(3):155-163

3. Barraclough B, Harris EC (2002) Suicide preceded by murder: the epidemiology of homicide-suicide in England and Wales 1988-92. Psychol Med 32:577-584

4. Foerster K (2009) Erweiterter Suizid. Ein problematischer Begriff? Nervenarzt 80:1078-1084

5. Lipp D (2018) Eine statistisch-deskriptive Auswertung der Suizide des Instituts für Rechtsmedizin München im Jahr 2010. Med Diss. LudwigMaximilians-Universität, München

6. Zimmermann MR (2017) „Erweiterte Suizide“ in Berlin zwischen 2005 und 2013 - Eine Obduktionsstudie. Med Diss. Charité - Universitätsmedizin, Berlin

7. Coid J (1983) The epidemiology of abnormal homicide and murder followed by suicide. Psychol Med 13:855-860

8. Eliason S (2009) Murder-suicide: a review of the recent literature. J Am Acad Psychiatry Law 37:371-376

9. Byard RW (2005) Murder-suicide: an overview. In: Tsokos M (Hrsg) Forensic pathology reviews, Bd. 3. Humana Press, Totowa, S337-347

10. Sehmer JM (2009) Hair dryer in the bath not necessarily fatal. Can Fam Physician 55(9):867
11. Primavesi R (2009) A shocking episode - care of electrical injuries. Can Fam Physician 55:707-709

12. Pollak S (1979) Erscheinungsformen des erweiterten Selbstmordes. Forensia 2:67-78

13. Byard RW, Knight D, James RA, Gilbert J (1999) Murder-suicides involving children: a 29-year study. Am J Forensic Med Pathol 20(4):323-327

14. Bossarte RM, Simon TR, Barker L (2006) Characteristics of homicide followed by suicide incidents in multiple states, 2003-04. Inj Prev 12(Suppl II):ii33-ii38

15. Palermo GB, Smith MB, Jenzten JM, Henry TE, KonicekPJ, Peterson GF, Singh RP, Witeck MJ (1997) Murder-suicide of the jealous paranoia type: a multicenter statistical pilot study. Am J Forensic Med Pathol 18(4):374-383

16. Campanelli C, Gilson T (2002) Murder-suicide in New Hampshire, 1995-2000. Am J Forensic Med Pathol 23(3):248-251

17. Rosenbaum M (1990) The role of depression in murder-suicide in couples involved and homicide. Am J Psychiatry 147(8):1036-1039

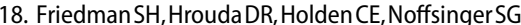
ResnickPJ (2005) Filicide-suicide: common factors in parents who kill their children and themselves. J Am Acad Psychiatry Law 33(4):496-504

19. Okumura Y, Kraus A (1996) Zwölf Patientinnen mit erweiterter Selbsttötung - Psychologie, Persönlichkeit, Motivation, Vorgeschichte und psychosoziale Konfliktsituation. Fortschr Neurol Psychiatr 64(5):184-191

20. Hellen $F$, Lange-Asschenfeldt $C$, Huckenbeck W, Hartung B (2014) Der ,erweiterte Suizid' Vollendete Homizid-Suizide unter psychopathologischen und kriminologischen Aspekten. Nervenarzt 85:1144-1150

21. Fishbain D, Rao V, Aldrich T (1985) Female homicide-suicide perpetrators: a controlled study. JForensic Sci 30(4):1148-1156

22. Resnick PJ (1969) Child murder by parents: a psychiatric review of filicide. Am J Psychiatry 126(3):73-82

23. Adelson L (1991) Pedicide revisited. Am J Forensic Med Pathol 12(1):16-26

24. Bockholdt B, Schneider V (2001) Death by electrocution in the bathtub. In: 4th Congress of the Baltic Medico-Legal Association Tartu, 22-25 August 2001

25. BonteW,Sprung R, HuckenbeckW(1986)Probleme bei der Beurteilung von Stromtodesfällen in der Badewanne. Rechtsmedizin 97:7-19

26. Lawrence RD, Spitz WU, Taff ML (1985) Suicidal electrocution in a bathtub. Am J Forensic Med Pathol 6(3):276-278

27. Pircher R, Pollak S, Vogt S, Epting T, Kramer L, Geisenberger D (2016) A special type of scald caused by prolonged exposure to slowly heated water. Forensic Sci Int 263:158-163

28. Rothschild MA, Bockholdt B, Schneider V (1997) Zur Problematik der suizidalen Strombeibringung in der Badewanne. Rechtsmedizin 7:131-134

29. Heckmann M, Brinkmann B, Fechner G (1990) Einfluß verschiedener technischer Parameter auf das Gefährdungspotential beim Stromunfall in der Badewanne. Beitr Gerichtl Med 48:329-333

30. Fechner G, Brinkmann B, Heckmann M (1990) Herzstromdichte als wichtigster biologischer Parameter bei Stromexposition in der Badewanne. Beitr Gerichtl Med 48:335-338

31. Logan JE, Walsh S, Patel N, Hall JE (2013) Homicidefollowed-by-suicide incidents involving child victims. Am J Health Behav 37(4):531-542
Springer Medizin Podcast

Medizin für Gesundheitsprofis

Der Podcast von SpringerMedizin.de geht seit Juli 2020 spannenden Fragen aus der Welt der Medizin nach immer freitags erweitert eine neue Folge das bereits bestehende OnlineAngebot.

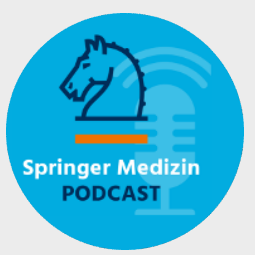

"Mit unserem

Podcast möch-

ten wir all jene ansprechen, die sich auf medizinische Themen in einer, gewissen

Flughöhe' einlassen möchten", erklärt Dr. Erik Heintz, Chefredakteur von SpringerMedizin.de das neue Format. „Gemeint sind damit Menschen, die sich nicht mit medizinischem Halbwissen zufrieden geben und gerne mehr wissen möchten. Daher legen unsere Redakteurinnen und Redakteure in München und Heidelberg vor allem auf den inhaltlichen Anspruch großen Wert. Unter Bezugnahme auf neue Studien, neue Erkenntnisse und praxisrelevantes Wissen bereiten wir jeden Podcast gründlich vor. Die Themen drehen sich um Gesundheit, Krankheit, Diagnostik, Therapie und Prävention, zu denen wir unsere Fachexpertinnen und Fachexperten aus verschiedenen medizinischen Fachgebieten hinzuziehen."

So entstehen abwechslungsreiche Folgen, die sowohl detailliert Leitlinien abarbeiten, der Evidenz von neuen Studienergebnissen auf den Grund gehen, aber auch die Hintergründe zu Erkrankungen und Therapien aufschlüsseln - sodass einerseits die ärztliche Praxis erleichtert und konkrete Tipps für die alltägliche Arbeit vermittelt werden, andererseits aber auch der Horizont über das eigene Fachgebiet hinaus erweitert werden kann.

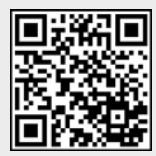

www.springermedizin.de/podcast 
Hier steht eine Anzeige.

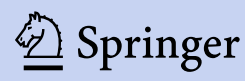

